\title{
Virtualization-support Cases in Engineering Education
}

\author{
Soler, José
}

Published in:

3rd International Congress on Engineering Education (ICEED 2011)

Link to article, DOI:

10.1109/ICEED.2011.6235348

Publication date:

2011

Link back to DTU Orbit

Citation (APA):

Soler, J. (2011). Virtualization-support Cases in Engineering Education. In 3rd International Congress on Engineering Education (ICEED 2011): Proceedings IEEE. https://doi.org/10.1109/ICEED.2011.6235348

\section{General rights}

Copyright and moral rights for the publications made accessible in the public portal are retained by the authors and/or other copyright owners and it is a condition of accessing publications that users recognise and abide by the legal requirements associated with these rights.

- Users may download and print one copy of any publication from the public portal for the purpose of private study or research.

- You may not further distribute the material or use it for any profit-making activity or commercial gain

- You may freely distribute the URL identifying the publication in the public portal

If you believe that this document breaches copyright please contact us providing details, and we will remove access to the work immediately and investigate your claim. 


\title{
Virtualization-support Cases in Engineering Education
}

\author{
José Soler, DTU Fotonik, Technical University of Denmark \\ joss@fotonik.dtu.dk
}

\begin{abstract}
The paper presents cases of applying hardware virtualization techniques as support for education activities in two different courses and a master thesis within the degree International MSc on Telecommunication Engineering at the Technical University of Denmark (DTU). The triggering problem is presented in each of the cases, together with the benefits and drawbacks of using virtualization to cope with it.
\end{abstract}

\section{Keywords - Virtualization, services, IMS, cloud.}

\section{INTRODUCTION}

The paper starts with a brief presentation of virtualization technology, its applicability realm and likely benefits as well as the chosen virtualization environment. Afterwards the three different cases where virtualization has been applied are presented. The first case deals with benefits for the University and course responsible, in terms of hardware cost reduction and reproducibility, while the second and third cases deal with benefits for the students, in terms of flexibility. Finally a critical analysis of the solutions and benefits in each of the cases is provided, as well as an outline for future experiences building on these initial ones.

\section{VIRTUALIZATION, IN SHORT}

Hardware virtualization provides a logical representation of a computer in software, a so-called virtual machine. A virtualization environment running on a host physical computer, offers an abstraction of the hardware physical resources of the host, which can be shared by multiple guest virtual machines running on that environment, each of them with its own virtual configuration regarding memory and storage capacity, resources configuration, operating system and network interfaces [1][2]. This is illustrated schematically in Figure 1.

Virtualization allows primarily avoiding the burden (physical, economical) imposed for the individual hardware requirements of a computer system. This allows for the creation of virtual deployments in order to test applications, or networking setups within a single host. The possibility of storing, keeping state and porting virtual machines, allows also for reproducibility of the virtual setups, which can be vital for security, backup or demonstration purposes.

Virtualization is currently the underlying technology allowing the so-called cloud and the provision of its different service models, i.e. Software as a Service

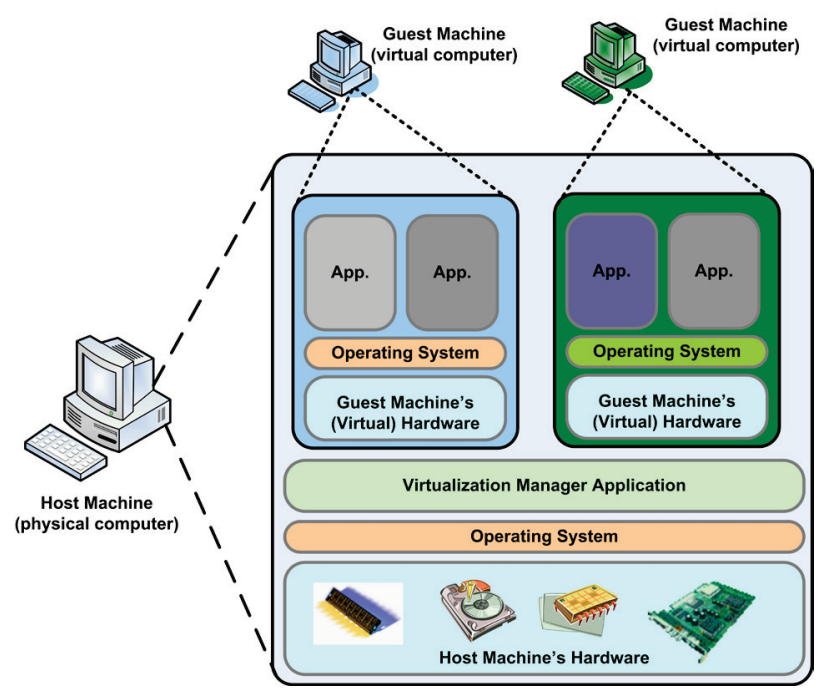

Figure 1. Hardware virtualization concept.

(SaaS), Platform as a Service (PaaS) and Infrastructure as a Service (IaaS) [3]

For the cases herein presented Oracle VM Virtual Box [4] was chosen as virtualization environment, due to its free availability, ease of use and support for multiple host and guest environments, which makes it ideal as a virtualization environment for students to experiment and learn with it. Furthermore, Virtual Box is an open source product, which opens for future possibilities in case that specific modification could be necessary for courses or student projects.

\section{AN INITIAL CASE: COURSE 34357}

DTU's course 34357, Advanced Telecommunications, deals with interaction of traditional telephony services with other, now current, IP services. The course starts dealing with issues of multimedia over IP and associated protocols (RTP, RTSP) and architectures for streaming applications. It continues with issues of Voice over IP and supporting protocols (H323, SIP), and moves on to interoperation between IP Telephony systems with conventional PSTN/SS7 telephony and IN supplementary telephony services. Different laboratory setups are used during the course as hands on experience for the students. One of them bases on traffic analysis of VoIP sessions mediated by an H323 Gatekeeper and a SIP proxy. The initial setup for these servers was based on hardware machines, under the control and responsibility of the University IT Support department. 
Accessibility issues to the physical machines and the need for coordination with the IT Support department led the responsible for the course, to look for alternative solutions for installation, running and maintenance of these servers. The final solution was to create a virtual deployment for the servers, as Linux guests on his Windows based desktop computer as a host (Intel Xeon e5520 CPU at 2,27 Ghz, with 12GB of RAM and 64-bit Windows 7 Enterprise OS). The only requirement, in regards to the IT support department, was to communicate to them the (virtual) MAC address of the guest virtual machine, so that it could get one of the universities internal DHCP-based IP addresses at start-up, when its network interface was in bridged mode, as per Virtual Box terminology. In this way the virtual machine could be associated with the existing domain names established for the lab VoIP services, within the University DNS.

From the point of view of students, the change from a physical setup to a virtual setup was seamless. No difference could be inferred from the traffic analysis or the final performance of the servers during the lab sessions. As a result, they were never aware that they were interacting against a virtualized server, nor they needed to. On the other hand, for the course responsible the difference was considerable: not only in terms of accessibility to the machines as it was the triggering problem, but mainly on additional benefits such as needless maintenance of hardware, possibility of backing up and porting a running and tested server to a different location, if necessary for demonstration needs or for offering the course at a different location or institution. Even in the case of a hardware failure of the hosting physical machine, having the virtual machine backed up (a mere data file), allows immediate recovery from any other computer where Virtual Box is installed, assuring in such a way a minimal disruption.

\section{A NEW CASE: COURSE 34358}

As a follow up for course 34357, the underlying idea of DTU's course 34358, Programming Services for Next Generation Networks, is to allow students to gain practical experience on programming SIP -based services, for environments such as Internet Multimedia Subsystem (IMS) [5].

The tools chosen for the course responsible as programming and deployment environments are based on Open Source software for Linux OSs, which made impossible to use the usual Databars in the campus for the lectures. The Databars at the University are lecture rooms equipped with 1 hardware personal computer per student place, where practical computer-based lectures and computer-based exercises are carried on. The impossibility to use these facilities for this course was motivated by the fact that these computers are only installed with Microsoft's OSs, without support for the tools chosen by the course responsible. Furthermore, due to security constraints, these computers at the Databars don't allow installations or modifications of software, besides an existing generic profile installation, which is reviewed once yearly. This made impossible for the course responsible to add the course requirements for a virtual setup on those computers.

As a solution, the course responsible decided to install and run the chosen service creation and deployment environment, on a Linux based virtual machine running as a Virtual Box guest machine on his host desktop computer (Intel Xeon e5520 CPU at 2,27 Ghz, with $12 \mathrm{~GB}$ of RAM and 64-bit Windows 7 Enterprise OS). The course responsible created and tested all the course examples there and afterwards ported and saved the final virtual machine. This final virtual machine with a running and tested environment, as well as with the created course examples on it, was provided to the students, who followed the course on a conventional lecture room, using their own laptop computers as hosts (the students are required a laptop to participate in the course).

The solution was partially successful. On the functional side the solution was successful since it allowed the student to work independently and on their own, even outside of the class or University environment. This allowed them also to replicate and test the development environment setup anywhere, based on Virtual Box capabilities to export and import virtual machines as a conventional data file. The drawback came from the heterogeneity of laptops used by the students, with different hardware elements which produced performance issues with laptops with non sufficient memory resources as compared to those of the host computer used by the course responsible for the initial testing of the setup.

\section{A STUDENT ON HIS OWN: ADAM'S THESIS}

As part of his work towards the MSc degree on Telecommunication Engineering, a student, Adam, had to investigate and evaluate differences between emulation and simulation strategies for testing service deployments in Next Generation telephony networks.

In order to do so, Adam had to setup multiple configurations of IMS and compare them and their functional performance when testing different SIP-based services (based on SIP-Servlets). This made impossible to have multiple dedicated physical computers in order to have different setups running simultaneously for comparative purposes or to introduce physical modifications to an existing setup in an easy as well as economical way.

The solution adopted was to provide the student with a relatively powerful computer with enough resources (Intel Xeon e5520 CPU at 2,27 Ghz, with 12GB of RAM and 64-bit Windows 7 Enterprise OS) as to behave as a host machine for Virtual Box based virtual setups.

With such environment Adam was able to test multiple IMS configurations within a single virtual machine as well as IMS setups based on networked virtual machines, thanks to the host-only network mode in Virtual Box. The setup gave him full flexibility to test and modify different elements and to save lot of time on installation, since he could just replicate an existing base reference virtual machine [6]. 


\section{LESSONS TO LEARN}

Virtualization provides an excellent environment for academics to experiment and test different setups which can be directly exposed and offered to students, at minimal effort and with a severe reduction on deployment time, costs and operative drawbacks.

While the reduction in equipment needs is clear, it is evident also that the supporting host machines need to be powerful enough in terms of memory and processing resources, so that the performance of the virtualized setups is not affected. This is especially important with multiple virtual machines running simultaneously on the same host machine.

It is also important to point out that, some services can be affected due to the fact of shared physical resources between virtual machines. For example in our experiences we had problems due to sharing the audio devices of the physical host with the virtual guest(s). It is important to understand the underlying mechanisms of the technology in order to trace and identify these issues, which can be sometimes misleading to students if they are not warned of them in advance.

\section{CONCLUSION}

The initial experiences applying Oracle's VM Virtual Box as virtualization technology for support of some of the educational activities at DTU have been successful, as presented in this paper. Benefits, both for students as well as for course responsibles and the institution have been described. It is our hope to continue using the technology in the following years and to build upon the experiences herein described. A permanent, virtual IMS lab, is likely the next step in this thread.

The open source nature of the chosen virtualization environment, Oracle's VM Virtual Box, opens also for possibility of additional work with it, in case that specific modifications or setups are necessary in the future.

\section{REFERENCES}

[1] Wolf C., Halter E., "Virtualization: from the desktop to the enterprise". Apress. Springer-Verlag. 2005. ISBN:1-59059-495-9.

[2] IBM Systems \& Technology Group. "Virtualization in Education. Whitepaper".IBM Corporation, 2007.

[3] Mell P., Grance T., "The NIST Definition of Cloud Computing". NIST Special Publication 800-145 (Draft). January 2011.

[4] http://www.virtualbox.org/, checked on the 16th June 2011.

[5] 3GPP TS 23.228. "IP Multimedia Subsystem (IMS). Stage 2, Release 11". V11.1.0. $3^{\text {rd }}$ Generation Partnership Project. June 2011.

[6] Galczynski A., "Comparative Evaluation of Simulation vs Emulation Environments for Testing Service Deployments in next generation Telephony Networks". DTU. October 2010. 